Pesq. Vet. Bras. 36(5):405-411, maio 2016

DOI: $10.1590 /$ S0100-736X2016000500009

\title{
Avaliação das alterações hemostáticas e do risco tromboembólico em cães com AHIM ${ }^{1}$
}

\author{
Lívia F. Moraes ${ }^{2 *}$, Regina K. Takahira², Marjorie de A. Golim³ e Márcia S. Baggio ${ }^{4}$
}

\begin{abstract}
Moraes L.F., Takahira R.K., Golim M.A. \& Baggio M.S. 2016. [Evaluation of hemostatic abnormalities and thromboembolic risk in dogs with IMHA.] Avaliação das alterações hemostáticas e do risco tromboembólico em cães com AHIM. Pesquisa Veterinária Brasileira 36(5):405-411. Departamento de Clínica Veterinária, Faculdade de Medicina Veterinária e Zootecnia, Universidade Estadual Paulista, Botucatu, SP 18618-970, Brazil. E-mail: liviafm@gmail.com

Immune-mediated hemolytic anemia (IMHA) is the most common cause of hemolytic anemia and the most prevalent immune-mediated disease in dogs, which is classified in primary or secondary. IMHA has been associated with hypercoagulability state and thromboembolism has been referred as the most common complication. The purpose of this study was to correlate the possibility of hemostatic abnormalities and the thromboembolic risk in IMHA and in anemias of other etiologies. 76 dogs were selected, all exhibiting hematocrit lower than $20 \%$ and at least one clinical sign commonly associated with IMHA or breed predisposition. These dogs were tested for IMHA by flow cytometry. Hemostatic tests (platelet count, PT, APTT, TT, AT, PDF and D-dimer) as well as CBC, reticulocyte count, blood parasite search in peripheral blood smear, Ehrlichia sp. and leptospirosis tests were performed in all dogs. 59 dogs were positive for IMHA. The thromboembolic risk was characterized by the presence of three or more changes in the tests of the hemostatic profile. $74.6 \%$ cases of IMHA were attributed to infectious diseases and was mostly associated with Ehrlichia sp. (88.6\%). $72.1 \%$ of dogs had thrombocytopenia and $57.6 \%$ had regenerative anemia with significantly higher values of metarubricyte and reticulocyte count. There were no significant changes between two anemic groups (positive and negative for IMHA). Anemic dogs had higher APTT mean value and lower AT and platelet counts mean values than the control group ( $\mathrm{p}<0.05)$. 25 dogs with IMHA and seven dogs without IHMA had thromboembolic state. The specificity of PDF was lower (30.2\%) than previous studies. The choice of the Ig class does not affect the diagnosis of IMHA. There were no correlation between the presence of antibodies bound to the RBC surface and the hemostatic tests, but there were a week correlation $(\mathrm{p}<0,05)$ between $\mathrm{Ht}(\%)$ and APTT $(\mathrm{r}=-0.2621)$, AT $(\mathrm{r}=0.4297)$ and platelets count ( $r=0.5349)$ values. Anemia is associated with hemostatic abnormalities and thromboembolic risk, however the immune-mediated nature of the disease was not the determining factor for this condition. In part, these changes can be attributed to infection diseases seen in both groups (positive and negative for IMHA). The similar thromboembolic risk evidenced in both groups of anemic dogs does not exclude the relationship of immune-mediated involvement, but alert to the possibility of thromboembolism in anemic dogs with Ehrlichia sp. (negative for IHMA). Therefore, anemic dogs with clinical signs associated with thromboembolic risk should be evaluated for this complication, today poorly
\end{abstract}

\footnotetext{
${ }^{1}$ Recebido em 6 de junho de 2015.

Aceito para publicação em 8 de março de 2016.

${ }^{2}$ Departamento de Clínica Veterinária, Faculdade de Medicina Veterinária e Zootecnia (FMVZ), Universidade Estadual Paulista (Unesp), Distrito de Rubião Júnior s/n, Botucatu, SP 18618-970, Brasil. *Autor para correspondência: liviafm@gmail.com

${ }^{3}$ Serviço de Citometria de Fluxo, Hemocentro de Botucatu, Hospital das
}

Clínicas, Faculdade de Medicina (FM), Universidade Estadual Paulista Júlio de Mesquita Filho (Unesp), Distrito de Rubião Júnior s/n, Botucatu, SP 16618-970.

${ }^{4}$ Laboratório de Hemostasia, Campus Universitário, Setor de Coagulação, Hospital das Clínicas, Faculdade de Medicina de Ribeirão Preto (FMRP), Universidade de São Paulo (USP), Monte Alegre, Ribeirão Preto, SP 14048-900, Brasil. 
investigated in clinical routine. The high frequency of hemostatic abnormalities supports the importance of an early, effective and more accurate diagnosis, along with a preventive strategy and treatment.

INDEX TERMS: Immune-mediated hemolytic anemia, thrombosis, hemostasia, Ehrlichia sp., dogs.

RESUMO.- A anemia hemolítica imuno-mediada (AHIM) é a causa mais comum dentre as anemias hemolíticas e a doença imuno-mediada de maior prevalência em cães, incluindo causas primárias e secundárias. As AHIM tem sido associadas a estados de hipercoagulabilidade, sendo o tromboembolismo a complicação mais comum. Este estudo teve como objetivo correlacionar as possíveis alterações hemostáticas e o risco tromboembólico nas AHIM e nas anemias por outras etiologias. Para tanto, foram selecionados 76 cães anêmicos (hematócrito $\leq 20 \%$ ) somados ao menos um sinal clínico comumente associado à AHIM ou possuir pré-disposição racial. Foram realizados os seguintes testes para os animais selecionados: teste de citometria de fluxo e avaliação do perfil hemostático (contagem de plaquetas, TP, TTPA, TT, AT, PDF e Dímeros D); além de hemograma com contagem de reticulócitos, pesquisa de hematozoários em sangue periférico, PCR para Ehrlichia sp. e sorologia para leptospirose. 59 cães foram positivos para AHIM. O estado tromboembólico foi caracterizado pela presença de alteração em três ou mais testes do perfil hemostático. 74,6\% casos de AHIM foram atribuídos às doenças infecciosas, sendo em sua maioria associados à Ehrlichia sp. (88,6\%). 72,1\% apresentaram trombocitopenia e $57,6 \%$ apresentaram anemia regenerativa com valores significativamente maiores de metarrubrícitos e contagem de reticulócitos. Não houve diferença estatística entre os grupos de cães anêmicos (positivos e negativos para AHIM). Os cães anêmicos apresentaram valores médios maiores de TTPA e menores de AT e contagem de plaquetas quando comparados aos cães saudáveis $(\mathrm{p}<0,05)$. 25 cães positivos e sete negativos apresentaram estado tromboembólico. A especificidade de PDF foi menor $(30,2 \%)$ quand o comparada outros estudos. A escolha da classe de Ig não interfere no diagnóstico de AHIM. Não houve correlação entre a presença de anticorpos ligados às hemácias e as variáveis hemostáticas, porém houve uma fraca correlação $(\mathrm{p}<0,05)$ entre o hematócrito e os valores de TTPA ( $\mathrm{r}=-0,2621)$, AT $(\mathrm{r}=0,4297)$ e contagem de plaquetas $(\mathrm{r}=0,5349)$. A anemia está associada a alterações hemostáticas e ao risco tromboembólico, no entanto a natureza imuno-mediada não é o fator determinante para esta condição. Em parte, estas alterações podem ser atribuídas ao quadro infeccioso observado em ambos os grupos (positivos e negativos para AHIM). 0 risco tromboembólico semelhante evidenciado nos dois grupos de cães anêmicos não exclui a relação do envolvimento imuno-mediado, mas atenta para uma possibilidade do tromboembolismo em cães anêmicos com Ehrlichia sp. (negativos para AHIM). Desta forma, cães anêmicos que apresentam sinais clínicos associados ao risco tromboembólico devem ser avaliados para tal complicação, hoje pouco investigada na rotina clínica. A alta frequência de alterações hemostáticas sustenta a importância de um diagnóstico precoce, efetivo e mais acurado, juntamente com uma estratégia de prevenção e tratamento.
TERMOS DE INDEXAÇÃO: Anemia hemolítica imuno-mediada, trombose, hemostasia, Ehrlichia sp., cães.

\section{INTRODUÇÃO}

A anemia hemolítica imuno-mediada (AHIM) é a causa mais comum dentre as anemias hemolíticas e a doença imuno-mediada de maior prevalência em cães (Mackin 2000, Quigley et al. 2001, McCullough 2003, Giger 2005). A AHIM é caracterizada pela destruição direta ou fagocitose de hemácias opsonizadas por imunoglobulinas como IgG ou IgM e/ou complemento. Os anticorpos podem ser direcionados às hemácias normais (AHIM primária ou idiopática) ou, ainda, às hemácias antigenicamente alteradas pela ação de medicamentos, neoplasias ou doenças infecciosas (leptospirose, babesiose, erliquiose canina), sendo esta última conhecida como AHIM secundária (Honeckman et al. 1996, Mackin 2000, McCullough 2003).

Há uma discordância em relação à prevalência das AHIM primárias e secundárias em cães. Tal discordância pode ser decorrente de uma maior prevalência real da AHIM secundária ou, então, da falta da realização de uma investigação diagnóstica mais detalhada em busca da causa base para a anemia em questão, pois o diagnóstico de AHIM primária é feito por exclusão (Scott-Moncrieff et al. 2001, Pereira 2006, Balch \& Mackin 2007). Um estudo realizado no interior de São Paulo evidenciou uma maior prevalência de AHIM secundária, discordando de resultados internacionais anteriores que afirmam a prevalência de AHIM primária (Pereira 2006).

0 tromboembolismo é a complicação mais comum nas AHIM e aproximadamente $50 \%$ dos cães apresentam estados de hipercoagulabilidade no momento do diagnóstico (Klag et al. 1993, McManus \& Craig 2001, Scott-Moncrieff et al. 2001, Carr et al. 2002). 0 tromboembolismo pulmonar tem sido atribuído como causa mais frequente de óbito em cães com AHIM, sendo encontrado em $80 \%$ dos casos em avaliação de necropsia (Carr et al. 2002). Um segundo estudo encontrou evidências de tromboembolismo em todas as necropsias realizadas em cães com AHIM (Weinkle et al. 2005).

Apesar da freqüência com que ocorrem os distúrbios hemostáticos em cães com AHIM e do fato de os eventos tromboembólicos serem frequentes causas de letalidade, o mecanismo para esta condição é desconhecido. Algumas hipóteses são levantadas para este estado de hipercoagulabilidade como o aumento da concentração de fatores pró-coagulantes, a diminuição da concentração de fatores anticoagulantes e fibrinolíticos, a presença de coagulação intravascular disseminada (CID), aumento da atividade plaquetária e a presença de anticorpos anti-fosfolipídeos (Scott-Moncrieff et al. 2001, Weinkle et al. 2005, Balch \& Mackin 2007).

Os sinais clínicos capazes de evidenciar um estado 
trombótico, na maioria das vezes, são inespecíficos ou ausentes. No entanto, a presença de fatores de risco como perda de proteína por nefropatia ou doença gastrointestinal, neoplasia, sepse, pancreatite, doenças imuno-mediadas ou vasculares e aumento dos níveis de corticóides consolida a suspeita de um possível estado trombótico, necessitando de uma investigação mais criteriosa (Nelson 2005).

Diversos autores relatam a incidência de CID em pacientes com AHIM entre $12 \%$ a $45 \%$ (Klag et al. 1993, Scott-Moncrieff et al. 2001, Carr et al. 2002, Balch \& Mackin 2007). A trombocitopenia intensa está associada ao alto risco de trombose em cães com AHIM, sendo que 90\% apresentam suspeita de CID (Carr et al. 2002).

Nos estudos de Scott-Moncrieff et al. (2001) e Carr et al. (2002) a coagulação intravascular disseminada (CID) foi diagnosticada em $45 \%$ e $32 \%$ dos casos, respectivamente, após uma avaliação completa para hemostasia, sendo que o primeiro estudo incluiu apenas os casos de AHIM primária. 0 diagnóstico confirmatório de CID pode ser difícil nas AHIM, pois grande parte das alterações hemostáticas também é observada nos animais apenas com tromboembolismo pulmonar (Balch \& Mackin 2007).

Neste trabalho objetivou-se buscar uma correlação das possíveis alterações hemostáticas e o risco tromboembólico nas anemias imuno-mediadas regenerativas ou arregenerativas nos casos de AHIM e nas anemias por outras etiologias, no município de Botucatu, SP.

\section{MATERIAL E MÉTODOS}

Foram selecionados 76 cães atendendo aos seguintes critérios: hematócrito inferior a $20 \%$, configurando um quadro de anemia regenerativa ou arregenerativa, sem históricos prévios de tratamento com corticóides ou transfusão sanguínea recente, além de apresentarem ao menos um sinal clinico (hepato ou esplenomegalia, icterícia, dispnéia, taquicardia, febre) ou laboratorial (esferócitos, auto-aglutinação, hiperproteinemia, hiperglobulinemia, blilirrubinúria) comumente associado à AHIM, ou ainda possuir pré-disposição racial (Cocker Spaniel, Old English Sheepdog, Doberman, Pinscher miniatura, Collie e Setter Irlandês). Foram excluídos os animais com outras causas conhecidas de anemia, como doença renal crônica, aplasia medular induzida por drogas como estrógeno ou agentes quimioterápicos e casos de verminose.

O diagnóstico de AHIM foi realizado por meio de teste de citometria de fluxo das hemácias dos cães anêmicos e os mesmos foram avaliados quanto ao perfil hemostático (tempo de protrombina - $\mathrm{TP}^{5 \mathrm{~A}}$, tempo de tromboplastina parcial ativada - $\mathrm{TTPA}^{\mathrm{A}}$, tempo de trombina - $\mathrm{TT}^{\mathrm{B}}$, antitrombina - $\mathrm{AT}^{\mathrm{B}}$, produto da degradação da fibrina - $\mathrm{PDF}^{\mathrm{B}}$ e Dímeros $\mathrm{D}^{\mathrm{B}}$ ) de acordo com as normas do fabricante, contagem de plaquetas, VPM, PDW (Hemascreen 18 - Ebram Produtos Laboratoriais Ltda, SP), pesquisa de hematozoários em sangue periférico, além dos testes para Ehrlichia sp. e leptospirose (apenas para os positivos para AHIM), pela técnica de reação em cadeia da polimerase (PCR) e sorologia, respectivamente. Para a avaliação do prolongamento de TP, TTPA e TT e diminuição da atividade de AT adotou-se um valor de corte com base na média dos valores encontrados em cães saudáveis (n=19) + 2 DP (desvio padrão). Para o estudo do perfil hemostático foi

\footnotetext{
5 A. Helena Laboratories.

B. Diagnostica Stago, Inc.

c. Bethyl laboratories, Montgomery, TX, USA.
}

formado um grupo controle composto por 26 cães saudáveis selecionados com base nos exames físico e laboratoriais, provenientes do canil do Hospital Veterinário da FMVZ/Unesp-Botucatu. 0 teste de citometria de fluxo das hemácias dos cães saudáveis também foi realizado para determinação do valor de corte para positividade para AHIM. Todos os cães anêmicos foram obtidos a partir da casuística do atendimento no Hospital Veterinário da FMVZ/Unesp-Botucatu, SP.

Para se constatar um estado tromboembólico os cães deveriam apresentar ao menos três das seguintes alterações: trombocitopenia, prolongamento do TP, TTPA ou TT, diminuição da atividade de antitrombina (AAT), aumento das concentrações de PDF e dímeros D. Para a avaliação da hemostasia terciária foram considerados animais realmente positivos os que apresentaram aumento nas concentrações de PDF e Dímeros D. Este critério de avaliação do risco tromboembólico já foi utilizado em diversos estudos anteriores que também associaram o risco tromboembólico com as alterações nos exames de hemostasia; bem como a diminuição da AAT (inferior a 30\%), que por si só, indica um risco iminente de tromboembolismo (Carr et al. 2002, Monreal 2003, Kuzi et al. 2010, Machida et al. 2010).

A CF foi realizada no equipamento FacScalibur (Becton Dickinson), utilizando-se o software CELLQuest. $100 \mu \mathrm{L}$ do concentrado de hemácias foram diluídos em 4,9mL de solução PBS. Esta solução foi centrifugada, em seguida foi removido o sobrenadante e acrescentou-se 4,9mL de solução PBS para uma nova lavagem e subseqüente centrifugação. Este procedimento foi realizado por mais duas vezes, obtendo uma solução de hemácias a 2\%. Para utilização na técnica de $\mathrm{CF}$, obteve-se uma solução de hemácias a 1\%. Foram utilizados anticorpos policlonais monoespecíficos caprinos $^{C}$ anti-IgG (anti-IgG de cão marcado com fluoresceína - FITC) e anti-IgM (anti-IgM de cão marcado com FITC). Foram utilizados três tubos, sendo o primeiro tubo, um tubo controle contendo apenas $100 \mu \mathrm{L}$ da solução de hemácias a $1 \%$, ao segundo e terceiro tubos foi adicionado $1 \mu \mathrm{L}(1,0 \mathrm{mg} / \mathrm{mL})$ de antiglobulina policlonal caprina anti-IgG ou anti-IgM de cão, respectivamente. Os tubos foram incubados por 20 minutos em ambiente escuro em temperatura ambiente. As hemácias foram novamente lavadas com 1,0mL de PBS. A leitura foi realizada imediatamente após a preparação da amostra.

A população de hemácias foi selecionada com base nos parâmetros FSC (forward scatter - tamanho) e SSC (side scatter - granulosidade), e a presença de IgG e IgM na superfície das células foi avaliada pela intensidade de fluorescência (FL1). Foram adquiridos 20.000 eventos para confirmação do diagnóstico de AHIM e classificação da classe de imunoglobulinas (IgG ou IgM) envolvidas.

A seleção da população de hemácias foi realizada por meio de "gate", desenhado com base nos parâmetros FSC e SSC, conforme recomendado na literatura (Harrus et al. 1996, Honeckman et al. 1996, Bateman et al. 1998). A utilização de "gate" é importante para excluir qualquer sinal oriundo de contaminação por plaquetas ou debris celulares (Harrus et al. 1996). Adotou-se um valor de corte para positividade com base na média das porcentagens de células marcadas dos cães controles +2 DP, baseado em estudos anteriores (Carr et al. 2002, Harrus et al. 1996); sendo consideradas positivas para AHIM amostras com fluorescência para IgG maior que $16,72 \%$ e para IgM maior que 9,56\% (Fig.1).

0 hemograma foi realizado no contador automático de células HEMASCREEN 18 (Ebran) e incluiu os seguintes parâmetros: eritrograma (hematócrito, contagem de hemácias, hemoglobina, VCM, CHCM, RDW), leucograma (leucócitos totais, valores relativos e absolutos), contagem de plaquetas, VPM e PDW.

Para a determinação da contagem de reticulócitos foi utilizada a técnica manual em esfregaços sanguíneos após coloração com 

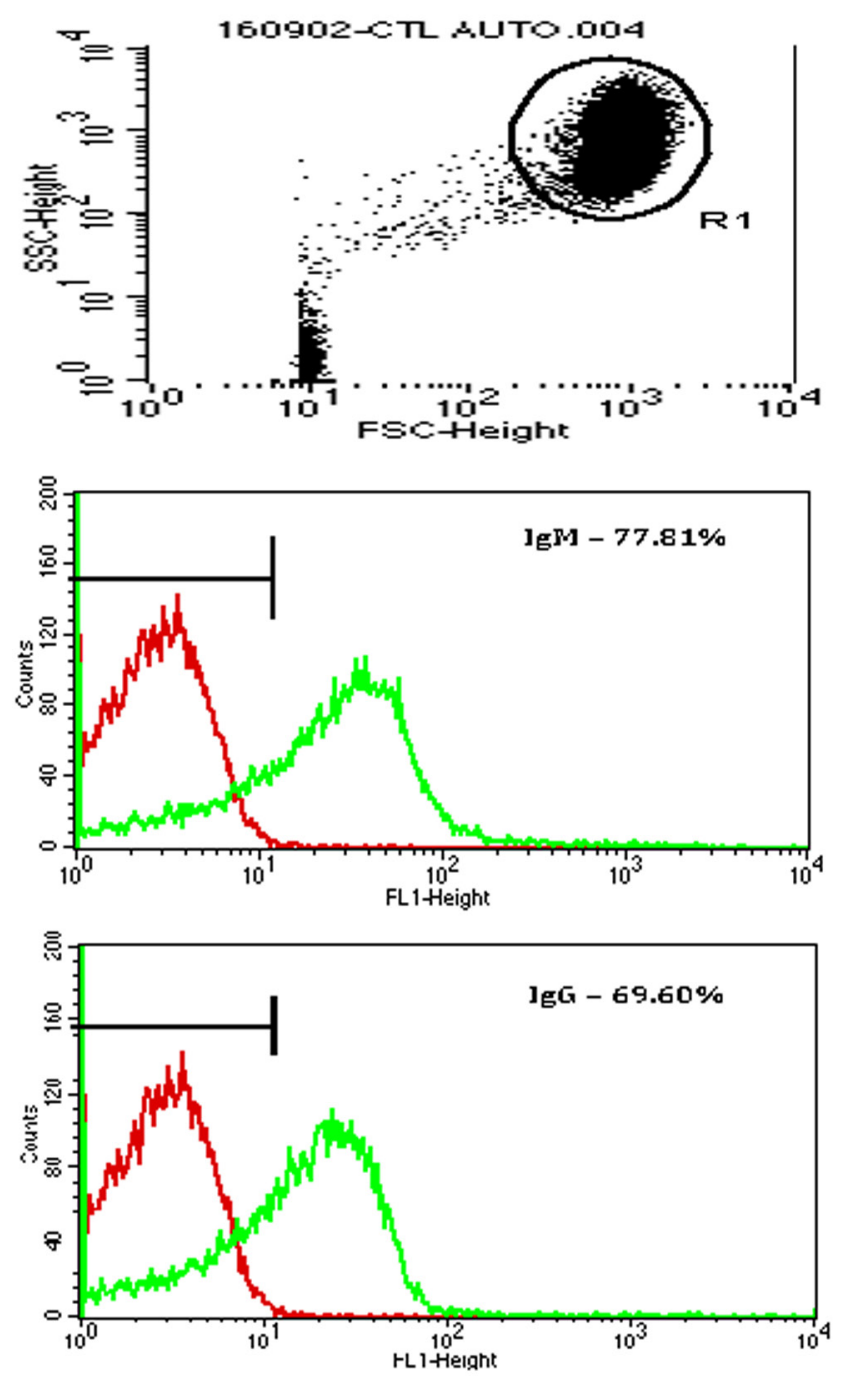

Fig.1. Quantificação das imunoglobulinas ligadas a eritrócitos por citometria de fluxo. Gate de hemácias baseado em Forward e Side Scatter. Em seguida, histogramas de IgM e IgG - curvas de teste positivo (verde) e auto controle (vermelho).

Novo Azul de Metileno e incubação em banho-maria a $37^{\circ} \mathrm{C}$ por 15 minutos.

Os testes de PCR, sorologia e pesquisa de hematozoários em esfregaço sanguíneo foram utilizados para classificação das AHIM em primária ou secundária. As AHIM primárias foram confirmadas nos casos negativos para estes testes e sem histórico de administração de medicamentos ou vacinas em um período de quatro semanas, neoplasia ou outras doenças infecciosas.

Este estudo foi aprovado pela câmara de ética em experimentação animal (CEEA) sob protocolo de número 165/2008.

Os resultados obtidos foram submetidos à análise descritiva para a avaliação da incidência das alterações hemostáticas, bem como da incidência da classe de imunoglobulina envolvida. Os resultados foram agrupados de acordo com os resultados encontrados na CF e quanto ao tipo de AHIM (primária ou secundária). A comparação das médias das variáveis entre os animais dos referidos grupos (positivos ou negativos para AHIM) foi analisada pelo teste t ou Mann-Whitney, de acordo com o tipo de distribuição da variável, no programa Sigmastat 3.1. Para as análises do perfil hemostático foi utilizado o teste de Kruskal-Wallis com post-hoc Dunn. A avaliação de correlação entre os resultados dos testes de hemos- tasia (variáveis quantitativas - TP, TTPA, TT, AT e contagem de plaquetas), citometria de fluxo e das variáveis hematológicas foi feita por meio do teste de Pearson. Todas as análises foram realizadas no programa BioEstat 5.0 e avaliadas ao nível de $5 \%$ de significância.

\section{RESULTADOS}

Dos 76 cães avaliados, 59 foram positivos para AHIM. Não houve predomínio de uma classe de Ig envolvida nas AHIM, pois 57 cães foram positivos tanto para IgG quanto para $\operatorname{IgM}$. Apenas um cão foi positivo somente para $\operatorname{IgM}(27,43 \%$ de fluorescência) e um outro positivo somente para IgG (34,63\% de fluorescência), sendo que nestes casos a AHIM foi detectada apenas pela técnica de CF.

As alterações clínico-laboratoriais mais comuns nos cães positivos incluíram esplenomegalia, icterícia, febre, auto-aglutinação, hiperglobulinemia e bilirrubinúria. A maioria dos cães não possuía raça definida e, dentro das raças predispostas à AHIM, quatro eram Cocker Spaniel e um Collie, sendo três casos de AHIM primária em cães da raça Cocker Spaniel.

$74,6 \%$ (44/59) dos casos de AHIM foram secundários a doenças infecciosas, em sua maioria associadas à Ehrlichia sp $(88,6 \%)$. Também foram diagnosticados casos associados à leptospirose, cinomose, babesiose e neoplasia. No grupo dos animais negativos $64,7 \%(11 / 17)$ foram positivos para Ehrlichia sp. Dentre as AHIM primárias (15 cães - 24,6\%, sendo oito fêmeas) 12 cães apresentaram anemia regenerativa e 10 auto-aglutinação.

As AHIM se caracterizaram por anemia regenerativa, macrocítica, hipocrômica e reticulocitose e, neste estudo, o grau de regeneração foi maior quando comparado com outras causas de anemias em cães (grupo negativo para AHIM), apesar da intensidade da anemia não apresentar diferença estatística entre os grupos positivos e negativos para AHIM. 57,6\% (34/59) dos cães positivos apresentaram anemia regenerativa. Para as variáveis do hemograma, os cães com AHIM apresentaram valores de metarrubrícitos e contagem de reticulócitos significativamente maiores $(\mathrm{p}<0,05)$ que os animais anêmicos negativos para AHIM. $10,2 \%$ dos cães com AHIM apresentaram esferocitose, $30,5 \%$ leucocitose e 39\% neutrofilia. A maioria dos cães negativos $(70,6 \%)$ apresentou uma anemia arregenerativa.

Os valores médios de plaquetas foram inferiores aos encontrados nas anemias por outras etiologias (grupo negativo para AHIM), apesar da ausência de diferença estatística entre os grupos de cães anêmicos. $72,9 \%$ dos cães positivos apresentaram trombocitopenia. A porcentagem de trombocitopenia no grupo dos cães negativos foi semelhante $(64,7 \%)$. Houve uma diferença estatística para VPM, sendo que os cães positivos apresentaram plaquetas menores (media 11,88 $\pm 2,93 ; \mathrm{p}=0,034$ ) quando comparados com aos cães negativos (media 13,78 $\pm 2,9$ ).

As variáveis TTPA, AT e contagem de plaquetas revelaram-se significativamente diferentes $(\mathrm{p}<0,05)$ em relação ao grupo controle, tanto para os cães positivos quanto para os negativos $(\mathrm{p}=<0,0001)$. Na comparação entre os grupos negativo e positivo para AHIM não houve alterações significativas (Quadro 1).

25 cães positivos para AHIM apresentaram diminuição 
da atividade de AT (AAT), sendo que dois apresentaram AAT $<30 \%$ e sete AAT $<60 \%$. Dentre os cães negativos, oito cães apresentaram diminuição da AAT, sendo que três apresentaram AAT $<60 \%$. (Quadro 2)

Para o TP houve diferença estatística apenas entre os cães negativos e saudáveis $(\mathrm{p}=0,0194)$ Não foram constatadas alterações significativas para o TT (Quadro 1).

No grupo dos cães positivos 46 cães apresentaram prolongamento do TTPA, nove do TP e três do TT (Quadro 2). 46 cães apresentaram aumento nas concentrações de PDF, sendo 19 cães com concentrações de $5-20 \mu \mathrm{g} / \mathrm{mL}, 10$ de 20 $40 \mu \mathrm{g} / \mathrm{mL}$ e 17 com concentrações de $\geq 40 \mu \mathrm{g} / \mathrm{mL}$ (Quadro 3). 18 animais obtiveram aglutinação em látex positiva

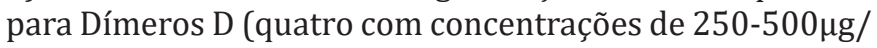
$\mathrm{mL}$; cinco entre $500-1000 \mu \mathrm{g} / \mathrm{mL}$, quatro de $1000-2000 \mu \mathrm{g} /$ $\mathrm{mL}$, dois de 2000-4000 $\mu \mathrm{g} / \mathrm{mL}$ e três com concentrações de $>4000 \mu \mathrm{g} / \mathrm{mL}$ ) (Quadro 4).

Dentre os cães negativos, 13 apresentaram prolongamento do TTPA, três do TP e três do TT (Quadro 2). 14 cães apresentaram aumento nas concentrações de PDF, sendo

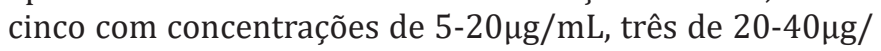
$\mathrm{mL}$ e seis com concentrações de $\geq 40 \mu \mathrm{g} / \mathrm{mL}$ (Quadro 3). Cinco cães obtiveram aglutinação em látex positiva para Dímeros D (dois com concentrações de $250-500 \mu \mathrm{g} / \mathrm{mL}$; um de $500-1000 \mu \mathrm{g} / \mathrm{mL}$ e dois com concentrações de $>4000 \mu \mathrm{g} /$ $\mathrm{mL}$ ) (Quadro 4).

Não houve diferença estatística para PDF e Dímeros D

Quadro 1. Médias, desvios- padrão e valor de p para TP, TTPA, TT, AT e contagem de plaquetas dos cães positivos para AHIM, negativos e cães saudáveis

\begin{tabular}{lcccc}
\hline & $\begin{array}{c}\text { Positivo para } \\
\text { AHIM }\end{array}$ & $\begin{array}{c}\text { Negativo para } \\
\text { AHIM }\end{array}$ & $\begin{array}{c}\text { Cães } \\
\text { Saudáveis }\end{array}$ & Valor de $\mathrm{p}$ \\
\hline TP (s) & $8,59 \pm 4,68^{\mathrm{ab}}$ & $8,88 \pm 4,40^{\mathrm{b}}$ & $6,73 \pm 1,28^{\mathrm{a}}$ & $\mathrm{p}=0,0194$ \\
TTPA (s) & $20,10 \pm 13,95^{\mathrm{a}}$ & $18,64 \pm 7,47^{\mathrm{a}}$ & $11,81 \pm 0,75^{\mathrm{b}}$ & $\mathrm{p}=<0,0001$ \\
TT (s) & $14,66 \pm 2,19$ & $15,70 \pm 3,64$ & $15,54 \pm 1,33$ & $\mathrm{p}=0,8899$ \\
AT (\%) & $97,96 \pm 35,04^{\mathrm{a}}$ & $96,88 \pm 35,37^{\mathrm{a}}$ & $133,08 \pm 20,81^{\mathrm{b}}$ & $\mathrm{p}=<0,0001$ \\
Plaquetas & $111.072 \pm$ & $138.994 \pm$ & $323.788 \pm$ & $\mathrm{p}=<0,001$ \\
(/ $\mu \mathrm{L})$ & $134.518^{\mathrm{a}}$ & $150.018,6^{\mathrm{a}}$ & $94.795,75^{\mathrm{b}}$ &
\end{tabular}

Médias seguidas de pelo menos uma letra em comum não diferem estatisticamente.

Quadro 2. Prolongamentos de TP, TTPA e TT e diminuição da atividade de AT em positivos e negativos para AHIM

$\begin{array}{llll}\text { TP TTPA } & \text { TT }\end{array}$

POS. $\quad 09 / 59(15,2 \%) \quad 46 / 59(77,9 \%) \quad 03 / 59(5,1 \%) \quad 25 / 59(42,4 \%)$ NEG. $\quad 03 / 17(17,6 \%) \quad 13 / 17(76,5 \%) \quad 03 / 17(17,6 \%) \quad 08 / 17(47 \%)$

Quadro 3. Concentrações de PDF ( $\mu \mathrm{g} / \mathrm{mL}$ ) em cães positivos e negativos para AHIM

\begin{tabular}{rrrr}
\hline Negativo & $5-20$ & $20-40$ & $\geq 40$ \\
\hline
\end{tabular}

POS. $\quad 13 / 59(22,0 \%) \quad 19 / 59(32,2 \%) \quad 10 / 59(16,9 \%) \quad 17 / 59(28,8 \%)$ NEG. $\quad 03 / 17(17,6 \%) \quad 05 / 17(29,4 \%) \quad 03 / 17(17,6 \%) \quad 06 / 17(35,3 \%)$ entre os cães anêmicos positivos e negativos para AHIM. $\mathrm{Na}$ avaliação do estado tromboembólico, 25 cães positivos $(42,3 \%)$ e sete cães negativos $(41,2 \%)$ atenderam aos critérios, apresentando as alterações laboratoriais hemostáticas compatíveis com o quadro.

Não houve correlação entre a presença de anticorpos ligados às hemácias e as variáveis hemostáticas, porém houve uma fraca correlação $(\mathrm{p}<0,05)$ entre o hematócrito e os valores de TTPA $(r=-0,2621)$, AT $(r=0,4297)$ e contagem de plaquetas $(r=0,5349)$.

\section{DISCUSSÃO}

A igual prevalência de IgG e IgM não privilegia a escolha da classe de imunoglobulina envolvida para o diagnóstico de AIHM pela técnica de CF.

Neste estudo os cães anêmicos positivos e negativos para AHIM apresentaram diferenças estatísticas em relação ao grupo controle para contagem de plaquetas, TP, TTPA, AT, PDF e Dímeros D. A maioria dos casos de anemia foi associada à Ehrlichia sp. 0 risco tromboembólico foi detectado tanto nos animais positivos quanto negativos para AHIM, demonstrando a importância da avaliação hemostática especialmente em áreas endêmicas para esta doença.

As alterações hematológicas e clínicas para cães com AHIM foram semelhantes às relatadas em outros estudos (Klag et al. 1993, Reimer et al. 1999, Carr et al. 2002, McCullough 2003, Goggs et al. 2008, Piek et al. 2008), incluindo anemia regenerativa, macrocítica, hipocrômica e reticulocitose com um grau de regeneração maior quando comparado com outras causas de anemias (grupo negativo), além de icterícia, febre, auto-aglutinação, hiperglobulinemia e bilirrubinúria. 74,6\% dos casos de AHIM foram atribuídos às doenças infecciosas, a maioria associados à Ehrlichia sp. (88,6\%). A presença de áreas endêmicas para diversas doenças infecciosas pode contribuir para a maior prevalência de AHIM secundária. Em relação aos cães negativos a maioria das anemias foram arregenerativas e 64,7\% foram positivos para Ehrlichia sp. As alterações hemostáticas associadas às AHIM primárias incluem trombocitopenia, diminuição da concentração do fibrinogênio e AT, prolongamento do TP, TTPA, TC e TT e aumento dos PDF e Dímeros-D (Feldman et al. 1999, Scott-Moncrieff et al. 2001). Não existem relatos na literatura que relacionem as alterações hemostáticas com as AHIM predominantemente secundárias. Portanto, este estudo alerta para estas mesmas alterações nas AHIM secundárias.

Diversos autores relatam a trombocitopenia associada à AHIM (Reimer et al. 1999, Scott-Moncrieff et al. 2001, Carr et al. 2002, Goggs et al. 2008). A trombocitopenia em cães com AHIM pode estar relacionada à concomitante destruição imuno-mediada das plaquetas, aumento do consumo devido à vasculite, processos inflamatórios generalizados, CID ou sequestro (Scott-Moncrieff et al. 2001).

Quadro 4. Concentrações de Dímeros $\mathrm{D}(\mu \mathrm{g} / \mathrm{mL})$ em cães positivos e negativos para AHIM

\begin{tabular}{ccccccc}
\hline & Negativo & $250-500$ & $500-1000$ & $1000-2000$ & $2000-4000$ & $\geq 4000$ \\
\hline POS. & $41 / 59(69,5 \%)$ & $04 / 59(6,8 \%)$ & $05 / 59(8,5 \%)$ & $04 / 59(6,8 \%)$ & $02 / 59(3,4 \%)$ & $03 / 59(5,1 \%)$ \\
NEG. & $12 / 17(70,6 \%)$ & $02 / 17(11,8 \%)$ & $01 / 17(5,9 \%)$ & $0 / 17$ & $0 / 17$ & $02 / 17(11,8 \%)$
\end{tabular}


Os valores médios de plaquetas foram inferiores aos encontrados nas anemias por outras etiologias (grupo negativo para AHIM). A porcentagem de trombocitopenia foi semelhante entre os grupos anêmicos. Neste estudo a trombocitopenia observada $(72,1 \%)$ provavelmente está relacionada à infecção por Ehrlichia sp, pois grande parte dos animais apresentaram PCR positivo para tal, sendo esta diminuição relacionada ao consumo devido à vasculite ou mesmo destruição imuno-mediada observada nesta doença (Nelson \& Couto 2009). A trombocitopenia intensa está associada ao alto risco de trombose em cães com AHIM (Carr et al. 2002). A porcentagem de cães com AHIM e trombocitopenia associadas foi semelhante a estudos anteriores (Scott-Moncrieff et al. 2001, Carr et al. 2002), no entanto foi observado um número maior de casos $(54,2 \%)$ com uma contagem de plaquetas inferior a $50.000 / \mu \mathrm{L}$, sendo esta associada ao aumento da mortalidade e risco de trombose (Scott-Moncrieff et al. 2001, Carr et al. 2002).

As diferenças estatísticas observadas nos animais anêmicos em relação ao grupo controle para contagem de plaquetas, TP, TTPA, AT, PDF e Dímeros D podem ser atribuídas à causa base predominante para a anemia em questão, ou seja, ao quadro infeccioso observado em ambos os grupos (Ehrlichia sp.). De fato, houve uma alteração significativa para os testes dos cães positivos para esta enfermidade, entretanto, o quadro imuno-mediado parece não ser o fator determinante para estas alterações, já que não foram observadas diferenças estatísticas entre os grupos positivos e negativos para AHIM. Portanto, as anemias associadas à erliquiose devem ser consideradas como fator de risco ou incluídas no quadro de alterações clínicas relacionadas às possíveis alterações hemostáticas.

Estudos anteriores apontam apenas alterações na função plaquetária de cães com erliquiose (Gaunt et al. 1990, Harrus et al. 1996, Brandão et al. 2006, Cortese et al. 2006), sendo este o primeiro relato de alterações hemostáticas em cães com erliquiose sem envolvimento imuno-mediado.

A diminuição da atividade de antitrombina (AAT) tem sido relacionada ao risco tromboembólico e prognóstico desfavorável. Esta diminuição está relacionada com um aumento do consumo, diminuição da produção ou perda (renal, gastrointestinal ou sangramentos). Para cães, acredita-se que há um risco iminente quando a AAT é $<30 \%$ e um risco elevado com AAT $<60 \%$. Nos casos de AHIM, a diminuição da AAT ocorre pelo aumento no consumo nos estados de hipercoagulabilidade (Kuzi et al. 2010), reafirmada neste estudo. Não foi possível afirmar uma relação de perda renal com os casos de diminuição da AAT, pois a mesma não foi avaliada na urina. A perda de AT pela urina também contribui para o estado de hipercoagulabilidade (Nelson \& Couto 2009).

Independentemente da causa, a hemólise é uma importante condição pró-coagulante. A liberação do estroma das hemácias durante a hemólise pode ser o desencadeador da coagulação intravascular disseminada (CID). A alteração das membranas dos eritrócitos e a presença de micro-partículas aumenta a conversão de protrombina em trombina e ativa a via intrínseca da cascata da coagulação, respectivamente. Fatores teciduais são produzidos e liberados pelos leucócitos quando as hemácias estão sensibilizadas por auto-anticorpos, sendo estes fatores de grande importância na CID. A presença de hemoglobina e heme, como produto da destruição dos eritrócitos durante a hemólise, estimula a expressão de moléculas de adesão pelas células endoteliais ocasionando a obstrução dos vasos, hipóxia e morte tecidual. A presença de hemoglobina livre também predispõe a estados de hipercoagulabilidade atribuída à ligação com o óxido nítrico (NO) e ao aumento da agregação plaquetária. NO é vital para a vasodilatação e atua como um potente inibidor da agregação plaquetária (Carr et al. 2002, Cappellini 2007).

A associação dos testes de hemostasia utilizados neste estudo (TTPA, PDF, Dímeros D e contagem de plaquetas) confere maior confiabilidade ao diagnóstico de um estado tromboembólico por determinar uma maior acurácia, sensibilidade e especificidade (Machida et al. 2010). Bateman et al. (1998) mencionam os testes de TP, TTPA, AT e contagem de plaquetas como sendo os mais indicados para o diagnóstico de CID, entretanto não incluindo a avaliação de PDF e Dímeros D.

Dentre os animais positivos para PDF, 28 cães com AHIM $(60,9 \%)$ e nove anêmicos negativos $(62,3 \%)$ apresentaram resultados negativos para Dímeros D. A possibilidade de resultados falsos positivos para PDF deve ser considerada. Considerando esta possibilidade, a especificidade de PDF foi bem menor $(30,2 \%)$ quando comparada com outros estudos anteriores que relatam a especificidade do teste de $72-90 \%$, semelhante para Dímeros D, mas este último possui maior sensibilidade (Caldin et al. 1998, Stokol et al. 2000, Boisvert et al. 2001). Os aumentos nas concentrações de PDF e Dímeros D associados confere maior especificidade e sensibilidade para o diagnóstico de um estado tromboembólico (Machida et al. 2010, Nelson \& Andreasen 2003). Outros estudos demonstram o uso promissor de Dímeros D para triagem de casos de CID e doença tromboembólica antes mesmo de evidências clínicas (Scott-Moncrieff et al. 2001, Griffin et al. 2003, Nelson \& Andreasen 2003). Uma concentração negativa para Dímeros D, de fato, pode excluir uma suspeita de tromboembolismo (Nelson 2005).

Este estudo alerta para a alta prevalência de AHIM, principalmente secundária relacionada à Ehrlichia sp em áreas endêmicas para esta doença infecciosa. Estas anemias são regenerativas e mais intensas quando comparadas com as anemias por outras etiologias e, em grande parte dos casos, associadas a uma trombocitopenia importante.

0 risco tromboembólico semelhante evidenciado nos dois grupos de cães anêmicos não exclui a relação do envolvimento imuno-mediado, mas atenta para uma possibilidade do tromboembolismo em cães anêmicos com Ehrlichia sp. A ausência de correlação entre a presença de anticorpos aderidos à superfície das hemácias e os resultados dos exames laboratoriais de hemostasia e a fraca correlação observada entre a intensidade da anemia e os indicadores de risco trombótico corroboram com essa afirmação.

Desta forma, cães anêmicos que apresentam sinais clínicos associados ao risco tromboembólico devem ser avaliados para tal complicação, hoje pouco investigada na rotina clínica. 
Agradecimentos.- À FAPESP pelo auxílio à pesquisa no 2008/10427-3.

\section{REFERÊNCIAS}

Balch A. \& Mackin A. 2007. Canine immune-mediated hemolytic anemia: treatment and prognosis. Comp. Cont. Educ. Pract. Vet. 29:230-238.

Bateman S.W., Mathews K.A. \& Abrams-Ogg Acg. 1998. Disseminated intravascular coagulation in dogs: review of the literature. J. Vet. Emerg. Critic. Care 8:29-45.

Boisvert A.M., Swenson C.L. \& Haines C.J. 2001. Serum and Plasma Latex Agglutination Tests for Detection of Fibrin(ogen) Degradation Products in Clinically Ill Dogs. Vet. Clin. Pathol. 30:133-136.

Brandão L.P., Hasegawa M.Y., Hagiwara M.K. \& Kohayagawa A. 2006. Platelet aggregation studies in acute experimental canine ehrlichiosis. Vet. Clin. Pathol. 35:78-81.

Caldin M., Fulanello T. \& Lubas G. 1998. Sensitivity and specificity of citrated plasma FDPs and D-dimer in the diagnosis of disseminated intravascular coagulation (DIC) in dogs. J. Vet. Intern. Med. 12:236. (Abstract)

Cappellini M.D. 2007. Coagulation in the pathophysiology of hemolytic anemias. Hematol. 1:74-78.

Carr A.P., Panciera D.L. \& Kidd L. 2002. Prognostic factors for mortality and thromboembolism in canine immune-mediated hemolytic anemia: a retrospective study of 72 dogs. J. Vet. Intern. Med. 16:504-509.

Cortese L., Pelagalli A., Piantedosi D., Mastellone V., Manco A., Lombardi P., Ciaramella P. \& Avallone L. 2006. Platelet Aggregation and Haemostatic Response in Dogs Naturally Co-infected by Leishmania infantum and Ehrlichia canis. J. Vet. Med. A., Phisiol. Pathol. Clin. Med. 53:546-548.

Feldman B.F., Madewell B.R. \& 0'Neill S. 1999. Disseminated intravascular coagulation: Antithrombin, plasminogen and coagulation abnormalities in 41 dogs. J. Am. Vet. Med. Assoc. 179:151-154.

Gaunt S.D., Baker D.C. \& Babin S.S. 1990. Platelet aggregation studies in dogs with acute Ehrlichia platys infection. Am. J. Vet. Res. 51:290-293.

Giger U. 2005. Regenerative anemias caused by blood loss or hemolysis, p.1886-1907. In: Ettinger S.J. \& Feldman E.C. (Eds), Textbook of Veterinary Internal Medicine. 6th ed. Elsevier Saunders, St Louis, Missouri. 2208p.

Goggs R., Boag A.K. \& Chan D.L. 2008. Concurrent immune-mediated haemolytic anaemia and severe thrombocytopenia in 21 dogs. Vet. Rec. 163:323-327.

Griffin A., Callan M.B., Shofer F.S. \& Giger U. 2003. Evaluation of a canine D-dimer point-of-care test kit for use in samples obtained from dogs with disseminated intravascular coagulation, thromboembolic disease, and hemorrhage. Am. J. Vet. Res. 64:1562-1569.

Harrus S., Waner T., Eldor A., Zwang E. \& Bark H. 1996. Platelet dysfunction associated with experimental acute canine ehrlichiosis. Vet. Rec. 139:290-293.

Honeckman A.L., Knapp D.W. \& Reagan W.J. 1996. Diagnosis of canine immune-mediated hematologic disease. Comp. Cont. Educ. Pract. Vet. 8:113-124.
Klag A.R., Giger U. \& Shofer F.S. 1993. Idiophatic immune-mediated hemolytic anemia in dogs: 42 cases (1986-1990). J. Am. Vet. Med. Assoc. 202:783-788.

Kuzi S., Segev G., Haruvi E. \& Aroch I. 2010. Plasma antithrombin activity as a diagnostic and prognostic indicator in dogs: a retrospective study of 149 dogs. J. Vet. Intern. Med. 24:587-596.

Machida T., Kokubu H., Matsuda K., Miyoshi K. \& Uchida E. 2010. Clinical use of D-dimer measurement for the diagnosis of disseminated intravascular coagulation in dogs. J. Vet. Med. Sci. 72:1301-1306.

Mackin A. 2000. Immune-mediated haemolytic anaemia, p.67-77. In: Day M.J., Mackin A. \& Littlewood J.D. (Eds), Manual of Canine and Feline Haematology and Transfusion Medicine. BSAVA, Gloucester. 320p.

McCullough S. 2003. Immune-mediated hemolytic anemia: understanding the nemesis. Vet. Clin. North Am. Small Anim. Pract. 33:1295-1315.

McManus P.M. \& Craig L.E. 2001. Correlation between leucocytosis and necropsy findings in dogs with immune-mediated hemolytic anemia: 34 cases (1994-1999). J. Am. Vet. Med. Assoc. 218:1308-1313.

Monreal L. 2003. Editorial: D-dimer as a new test for the diagnosis of DIC and thromboembolic disease. J. Vet. Intern. Med. 17: 757-759.

Nelson O.L. 2005. Use of the D-dimer assay for diagnosing thromboembolic disease in the dog. J. Am. Anim. Hosp. Assoc. 41:145-149.

Nelson O.L. \& Andreasen C. 2003. The utility of plasma D-dimer to identify thromboembolic disease in dogs. J. Vet. Intern. Med. 17:830-834.

Nelson R.W. \& Couto C.G. 2009. Small Animal Internal Medicine. 4th ed. Mosby, St Louis, Missouri. 1504p.

Pereira P.M. 2006. Avaliação de métodos diagnósticos (citometria de fluxo, teste da antiglobulina direta, auto-aglutinação, presença de esferócitos) e achados clinico-laboratoriais na anemia hemolítica imuno-mediada em cães. Tese de Doutorado em Medicina Veterinária, Clínica Médica, Faculdade de Ciências Agrárias e Veterinária, Universidade Estadual Paulista "Júlio de Mesquita Filho" - Unesp, Jaboticabal, SP. 92p.

Piek C.J., Junius G., Dekker A., Schrauwen E., Slappendel R.J. \& Teske E. 2008. Idiopathic immune-mediated hemolytic anemia: treatment outcome and prognostic factors in 149 dogs. J. Vet. Intern. Med. 22:366-373.

Quigley K.A., Chelack B.J., Haines D.M. \& Jackson M.L. 2001. Application of a direct flow cytometric erythrocyte immunofluorescence assay in dogs with immune-mediated hemolytic anemia and comparison to the direct antiglobulin test. J. Vet. Diagn. Invest. 13:297-300.

Reimer M.E., Troy G.C. \& Warnick L.D. 1999. Immune-mediated hemolytic anemia: 70 cases (1988-1996). J. Am. Anim. Hosp. Assoc. 35:384-391.

Scott-Moncrieff J.C., Treadwell N.G., McCullough S.M. \& Brooks M.B. 2001. Hemostatic abnormalities in dogs with primary immune-mediated hemolytic anemia. J. Am. Anim. Hosp. Assoc. 37:220-227.

Stokol T., Blue J.T. \& French T.W. 2000. Idiopathic pure red cell aplasia and non-regenerative immune-mediated anemia in dogs: 43 cases (19981999). J. Am. Vet. Med. Assoc. 216:1429-1436.

Weinkle T.K., Center S.A., Randolph J.F., Warner K. L., Barr S.C. \& Erb H.N. 2005. Evaluation of prognostic factors, survival rates, and treatment protocols for immune-mediated hemolytic anemia in dogs: 151 cases (1993-2002). J. Am. Vet. Med. Assoc. 226:1869-1880. 